\title{
Caring for patients with cancer histories in community-based primary care settings: a survey of primary care physicians in the southeastern US
}

Mary Ann Burg Department of Community Health and Family Medicine, University of Florida, FL, USA, Kathryn Grant Women's Health Research Center, University of Florida, FL, USA and Robert Hatch Department of Community Health and Family Medicine, University of Florida, FL, USA

As the numbers of cancer survivors grow, community-based primary care physicians are playing a larger role in their care. The goal of this pilot study was to begin to identify the problems and challenges faced by primary care physicians who treat patients with cancer histories. A mail survey was undertaken of 141 community-based primary care physicians in the Southeastern US that addressed physician characteristics, numbers and types of cancer patients treated, problems faced by these patients and challenges physicians face when caring for these patients. These physicians reported that on average $10 \%$ of their primary care patients had a history of cancer. The most frequent types of cancers seen by these physicians were skin, breast and prostate cancer, but there was a broad range of other cancers seen in their practices. They reported that patients and family members' fears and emotional problems were frequently encountered problems. Side-effects of cancer and its treatments and limited access to supportive care services were also reported as frequent problems for the cancer patients they treated. The most challenging aspect of caring for patients with cancer histories they reported was promoting cancer-related risk reduction behaviours. Primary care physicians play a large and important role in the care of cancer survivors. They encounter a number of problems when providing this care, including patient fears and access to supportive care. These findings may be used to guide educational and policy initiatives aimed at improving the care of cancer survivors.

Key words: cancer; cancer survivors; community; physician; primary care; surveillance; survivor

\section{Introduction}

The numbers of cancer survivors are increasing as early detection and cancer treatment improves. In 1999 there were almost 10 million cancer survivors

Address for correspondence: Mary Ann Burg, Women's Health Research Center, Box 100171, University of Florida, Gainesville, FL 32610 0171, USA. Email: burg@vpha.ufl.edu Prior Presentation: North American Primary Care Research Group (NAPCRG) 32nd Annual Meeting, Orlando, Florida, USA, 10 October 2004 in the US: site-specific five-year survival rates for the most prevalent cancers are 98.8 for prostate cancer, 86.4 for breast cancer, 61.7 for colon cancer and 15.0 for lung cancer (Jemal et al., 2004). Due to these improving survival rates, a large portion of cancer care now involves post-treatment care. Unfortunately, many cancer survivors are experiencing 'follow-up fallout', that is, they are not getting adequate medical surveillance and follow-up of cancer-related issues (Wang, 2002).

Increasingly, community-based primary care providers are being called on to serve as the 
'medical home' for cancer survivors and to respond appropriately to their unique sets of needs (Brotzman and Robertson, 1998; Centers for Disease Control, 2004; President's Cancer Panel, 2004). Delivering appropriate care to patients with a history of cancer involves a broad set of competencies including: knowledge of evidence-based cancer-specific surveillance guidelines to detect recurrent cancers; knowledge of genetic susceptibility to cancer of both the patient and the family; promotion of cancer-related risk reduction behaviours; monitoring for and managing cancer treatment complications; dealing with psychosocial problems related to the cancer experience; attention to cancer- and noncancer-related chronic and emergent health problems; and helping patients negotiate the cancer specialty care system (Kattlove and Winn, 2003).

To date, very little is known about post-treatment cancer care in the community health care sector. The purpose of this study was to conduct a pilot study of the types and extent of problems and challenges faced by community-based primary care physicians in caring for these individuals. We were particularly interested in detecting any association between practice location and the types of challenges faced by providers, since there is evidence that rural providers face more challenges in helping cancer survivors access health care resources (Liff et al., 1991; Howe et al., 1992). It was also hoped that these survey findings might yield useful information for developing relevant continuing medical education programs and content for medical school curriculum.

\section{Methods}

Human subjects' approval for this study was granted for by our University Institutional Review Board in March 2004.

This study data comes from a survey of $141 \mathrm{com}$ munity-based primary care physicians (family medicine, general medicine and paediatrics) who practice in rural and nonrural settings in North Florida. The physicians in this sample specifically are those who serve as preceptors to first-year medical students at the University of Florida during their two-week required course in community-based primary care.
Surveys were sent by mail to these 141 physicians during the months of April and May of 2004. The surveys were sent three times to maximize response rates (Dillman, 1978). Anonymity was maintained by including no items in the survey that could specifically identify the responding physician. All physicians who received the survey were asked to send back a postcard by separate mail to indicate whether they had completed the survey or if they refused to complete the survey. The survey instrument titled 'Meeting Needs of Patients with Cancer' was a two-page self-administered survey, including sociodemographical information, practice characteristics, numbers and types of cancer patients seen by the physicians, their perceptions of the needs of the patients with cancer histories they see in their practice, their evaluation of the degree of difficulty these patients have in meeting these needs, and their evaluation of the degree of difficulty they had as providers in meeting the unique needs of patients with cancer histories.

Descriptive statistics were used to explore respondent characteristics and to examine the distribution of responses to the survey questions. To examine associations between physician characteristics and their reporting of the problems their patients with cancer histories face, Likert-scale responses were re-coded into two-level variables (where responses of 'never problematic', 'seldom problematic' and 'sometimes problematic' were collapsed into 'little or no problem,' and responses of 'often problematic' and 'always problematic' were collapsed into 'some problem'). The same process of recoding was performed on the Likert-scale responses to questions about challenges the physicians face in caring for cancer survivors ('never a challenge,' 'seldom challenging' and 'sometimes challenging' compared to 'often challenging' and 'always challenging'). Chisquare analyses were used to explore statistically significant associations between physician characteristics (specialty, gender and practice location) and their perceptions of cancer care.

\section{Results}

\section{Response rate and respondents' characteristics}

Of the 141 physicians in the original sample, one was no longer in practice, six had incorrect addresses and one physician was incorrectly identified as a primary care physician. Of the remaining 133 
Table 1 Provider and practice characteristics

\begin{tabular}{|c|c|c|}
\hline & Frequency & Per cent \\
\hline \multicolumn{3}{|l|}{ Specialty } \\
\hline Family medicine & 36 & 55.4 \\
\hline General internal medicine & 18 & 27.7 \\
\hline Paediatrics & 11 & 16.9 \\
\hline \multicolumn{3}{|l|}{ Years post-training } \\
\hline $1-9$ years & 12 & 18.7 \\
\hline $10-19$ years & 33 & 51.6 \\
\hline 20 years or more & 19 & 29.7 \\
\hline \multicolumn{3}{|l|}{ Gender } \\
\hline Female & 17 & 29.8 \\
\hline Male & 40 & 70.2 \\
\hline \multicolumn{3}{|l|}{ Type of practice } \\
\hline Solo & 25 & 38.5 \\
\hline Group & 29 & 44.6 \\
\hline Other ${ }^{\mathrm{a}}$ & 11 & 16.9 \\
\hline \multicolumn{3}{|l|}{ Practice location } \\
\hline Rural & 23 & 36.5 \\
\hline Nonrural & 40 & 63.5 \\
\hline \multicolumn{3}{|l|}{ Average monthly patient volume } \\
\hline $1-400$ & 30 & 46.9 \\
\hline 400 & 34 & 53.1 \\
\hline
\end{tabular}

${ }^{\mathrm{a}}$ Includes public health department, hospital outpatient and 'other'

physicians in the sample, 26 sent back cards indicating they did not want to participate (19\%), and 42 did not respond to any of the three mailings and did not send back a card (32\%). The final response rate was $50 \%(66 / 133)$.

Family medicine physicians made up the majority of respondents followed by general internal medicine and paediatric physicians (Table 1). The average time since completion of clinical training for these respondents was 16 years. The majority of the respondents were male. Respondents were diverse in regards to where they practised and type of practice setting: approximately one-third practised in rural areas, and the majority of the respondents worked in either a group practice or solo practice; the remainder worked in public health or community-based clinics and hospital outpatient settings. The volume of monthly patient visits for these primary care practices ranged from 100 to 1500 per month, and averaged 461 per month.

\section{Volume and types of cancers seen in primary care settings}

Survey respondents were asked to estimate the volume and types of cancer histories they saw in
Table 2 Respondents' estimates of cancer survivors treated (per cent of patients seen in past year)

\begin{tabular}{lcc}
\hline $\begin{array}{l}\text { Estimated per cent } \\
\text { ranges }\end{array}$ & Frequency & Per cent \\
\hline $0.5-5$ & 22 & 35.5 \\
$6-10$ & 18 & 29.0 \\
$11-15$ & 9 & 14.5 \\
15 & 13 & 21.0 \\
\hline
\end{tabular}

\section{Skin cancer (including melanoma) \\ Breast cancer \\ Prostate cancer \\ Colon and rectal cancer \\ Lung \\ Female reproductive system cancer \\ Lymphoid cancers \\ Leukaemia and AML \\ Head and neck \\ Kidney and urological \\ Pancreatic cancer \\ Liver and biliary tree \\ Bone and soft tissue sarcomas \\ Brain and CNS cancer \\ Other ${ }^{\mathrm{a}}$}

a Includes appendreal, carcinoid, clear cell carcinoma, myeloma and multi-myeloma, gastric and peritoneal

Figure 1 Types of cancer seen in patients of communitybased primary care providers, in order of frequency reported

their practices. Specifically, they were asked 'Can you give me an estimate of the per cent of patients you saw in the past year who were diagnosed with cancer sometime in their lives'. On average, the physicians reported that $10 \%$ of their patients had a history of cancer (Table 2);35\% reported that more than $10 \%$ of their patients had a history of cancer. Physicians were also asked to list the types of cancers and estimate the total number of patients with specific types of cancers they had seen in the past year. The types of cancers are listed in order of the frequency physicians reported seeing them among their patients (Figure 1). Skin cancer was far the most frequent cancer seen in these primary care practices. Respondents' estimates of other prevalent 
Table 3 Per cent of physicians who encounter specific problems faced by cancer patients in their practice ${ }^{a}$

Specific problems

Patient's fear, anxiety or depression

Family problems with coping

Pain

Maintaining employment

Chemotherapy side-effects

Radiation side-effects

Accessing care giving resources

Accessing counselling services

Advice on use of complementary therapies

Distance to a cancer provider

Choosing a cancer provider

Advice on use of herbs and supplements

Sexual side-effects

Accessing support groups

Paying for cancer treatment

Lymphedema

Accessing follow-up care

Choosing type of cancer treatment

a Per cent of physicians who responded 'often' or 'always' a problem

types of cancers seen in their practice in order of reported frequency included breast, prostate, colon, lung and reproductive system cancers. The remaining cancers listed in Figure 1 were reported with much lower frequencies.

\section{Problems faced by cancer patients and barriers to getting needs met}

Physician respondents were asked to rate the frequency with which they encountered 18 specific problems faced by patients with histories of cancer (Table 3). Cancer survivors and their families' emotional problems were the most frequently encountered problems: specifically patients' 'fear, anxiety or depression' and 'family problems with coping'. They also reported patient problems with maintaining employment after cancer. Cancer and cancer treatment side-effects, especially pain, were also seen as problematic for patients by over onethird of the physicians. Patients' problems accessing supportive care resources (i.e., care-giving resources, counselling services and advice on complementary therapies) were reported by approximately one-fifth of the physicians.

Problems seen with less frequency by these physicians were barriers to specialty cancer care
Table 4 Per cent of physicians who typically experience specific challenges in caring for patients with cancer histories ${ }^{\mathrm{a}}$

\begin{tabular}{ll}
\hline Type of challenge & Per cent \\
\hline $\begin{array}{l}\text { Promoting cancer-related risk reduction } \\
\text { Attending to patients' and families fears } \\
\text { of recurrence }\end{array}$ & 48.3 \\
$\begin{array}{l}\text { Knowledge and capacity to use cancer } \\
\text { surveillance data }\end{array}$ & 21.9 \\
$\begin{array}{l}\text { Assessing and treating delayed } \\
\text { complications of cancer }\end{array}$ & 21.7 \\
$\begin{array}{l}\text { Assisting patients with advice and } \\
\text { referrals for recurrences }\end{array}$ & 18.3 \\
$\begin{array}{l}\text { Assisting terminal patients with } \\
\text { palliation and support }\end{array}$ & 16.4 \\
$\begin{array}{l}\text { Communication with oncologists caring } \\
\text { for my patients }\end{array}$ & 16.1 \\
\hline
\end{tabular}

a Per cent of physicians who responded 'often' or 'always' a challenge

(i.e., choosing types of cancer treatment, choosing a cancer provider), and access barriers (distance to cancer providers, paying for treatment and accessing follow-up care).

\section{Challenges to providing care to cancer survivors in primary care settings}

Physicians were asked to rate the level of challenge they typically faced in certain aspects of caring for cancer patients. Far the most challenging aspect of care for these physicians was promoting cancer-related risk reduction behaviours with patients; almost one-half of the physicians viewed this as particularly challenging (Table 4). Approximately one-quarter of the respondents reported feeling challenged in attending to patients' and families' fear of recurrence. One-fifth of the respondents were also challenged in the areas of knowledge of and capacity to use cancer surveillance data and assessing and treating delayed complications of cancer. Few of the physicians experienced challenges in communication with their patients' oncologists, in assisting terminal patients with palliation and support, and in providing advice and referrals to patients with recurrences.

There were no statistically significant differences in physicians who practised in rural vs. nonrural areas in terms of their reported challenges in caring for patients with cancer histories. There were also no significant associations between any other 
practice or physician characteristics, and reported challenges to caring for cancer survivors.

\section{Discussion}

This study, though limited in its generalizability, is the first known survey of community-based physicians' views on the problems and challenges of caring for patients with cancer histories in primary care. Our findings indicate that physicians are aware of the various physical, emotional and social problems faced by the many cancer patients they serve in their own communities; and they perceive some important, though often under-recognized challenges in providing quality follow-up cancer care to their patients.

Much of cancer care takes place outside of cancer centres, hospitals and oncology practices. According to US national data, approximately $15 \%$ of cancer care visits occur in primary care settings (Institute of Medicine and Commission on Life Sciences, 1999). The respondents to this survey reported having substantial numbers of patients with a variety of cancer histories in their practice. Previous research suggests that primary care physicians may under-estimate the number of patients with cancer histories they treat since there is evidence that some cancer histories, particularly paediatric cancer histories, are not always identified in primary care settings (Kadan-Lottick et al., 2002).

Providing quality care to patients with cancer histories in community-based primary care settings is complicated by the unique and wide ranging types of problems survivors experience. Although the majority of the physicians we surveyed believed it was particularly challenging to provide cancer risk reduction information, attend to survivor's emotional problems, and to conduct appropriate surveillance for recurrences, there is evidence for the competency of primary care physicians in these areas. A randomized-controlled trial compared follow-up of breast cancer patients between primary care settings and hospital outpatient settings in the UK. This analysis showed no delay in diagnosing recurrence and no differences in patients' anxiety or quality of life for those patients followed by primary care physicians (Grunfeld et al.,1996). The same authors examined relative costs of the two schemes for follow-up care and found that the primary care physicians actually ordered more diagnostic tests than the specialists, but that costs to the patients were lower in primary care (Grunfeld et al., 1999).

All primary care providers, regardless of their specialty or practice setting, can take an active lead in the routine management of the health care needs of cancer survivors. In terms of surveillance for cancer recurrence, current post-treatment cancer surveillance guidelines are less intensive than previously published guidelines, and require minimal routine surveillance methods that are for the most part compatible with primary care practice (Smith et al., 1999; Anthony et al., 2004). For example, for breast cancer surveillance the American Society of Clinical Oncology recommends a monthly breast self-exam, annual mammography, careful history and physical examination every three to six months for three years, then every six to 12 months for an additional two years, then annually (Smith et al., 1999).

The most prevalent short-term cancer-related side-effects reported in the literature are pain, fatigue and depression; long-term and late effects include secondary cancers, cognitive changes, cardiorespiratory dysfunction, sexual dysfunction and infertility, fatigue, menopausal symptoms, and mood disorders (Aziz, 2002; Dow, 2003). The physicians in this sample identified pain, and sideeffects of radiation and chemotherapy as problematic for their patients with cancer histories.

Reported prevalence rates for pain vary broadly, from $14 \%$ to $100 \%$, and most studies have focused on patients in active treatment for cancer or on patients with advanced cancer not on longterm survivors (Carr et al., 2002). A prospective study of post-treatment, disease-free head and neck cancer patients found that $48 \%$ of patients experienced pain at diagnosis, and $25 \%$ still reported pain at 24 months (Chaplin and Morton, 1999). Types of pain in chronic cancer pain sufferers include soft tissue, bone, visceral or neuropathic pain primarily (Petzke et al., 1999; Davis and Walsh, 2004). Pain is believed to be generally undertreated in cancer survivors. The diagnosis of pain is complicated by the reluctance of many patients to complain of pain and fears of opioid addiction, and these beliefs may be especially relevant in underserved minority patients (Anderson et al., 2002). Other barriers to appropriate pain management include inadequate training on standards of 
care in managing cancer pain, lack of time and resources to address pain, and concern about regulatory sanctions for overuse of opioids (National Institutes of Health, 2002). The World Organization of Health (WHO) has published a pain management guideline involving an evidence-based threestep analgesic ladder approach which can be useful for chronic cancer pain regardless of the aetiology (World Health Organization, 1996).

The common cancer-related side-effects are complex, often subjective experiences. They are hard to measure, and they often overlap with each other and with common symptoms of other chronic illnesses. These lingering and sometimes delayed effects of cancer treatments can vary widely from patient to patient; yet they contribute in a large part to the quality of life of cancer survivors and thus require more refined clinical attention and a broad spectrum of potential interventions. Lacking clear evidence for what side-effects to anticipate, physicians are faced with ambiguity in appropriate monitoring, diagnosing and treating the consequences of cancer treatment.

Our data did not provide support for the hypothesis that physicians in rural practices face more challenges in caring for patients with cancer histories than physicians in nonrural areas. It is interesting that overall, the physicians in rural practices seemed to encounter fewer challenges than the physicians in nonrural practices. Since our sample was very small, we cannot draw any conclusions about the rural/nonrural differences in caring for cancer survivors. We plan to follow up this pilot study with some qualitative data collection from community-based primary care physicians to explore more closely some of the challenges faced in providing care to cancer survivors and to examine specific geographical issues in accessing specialty and supportive care.

Published physician response rates for mail surveys are lower on average than non-physicians, specifically $54 \%$ compared to $68 \%$ (Asch et al., 1997). Although our survey generated a response rate close to the published average, it is important to note that our survey results are not generalizable to all community-based primary care physicians since we did not use a representative sampling procedure. It is possible that responders had higher numbers of cancer patients in their panels then non-responders, and were thus more highly motivated to complete the survey. However, we had no method of examining this potential source of bias. There was no statistically significant difference between in proportion of respondents vs. nonrespondents who practised in rural areas. However, we also had very limited power for examining differences between rural and nonrural respondents, and thus our results of no differences found in challenges to care based on practice location must be viewed with caution and examined in larger samples. Finally, the physicians who were surveyed were also a select group in that they were originally selected to meet the requirements of having the potential for being good teachers, having a setting conducive to student learning in primary care (and thus solo practices are over-represented), and they agreed to take first-year medical students into their offices for two and half weeks each year. In the future we would like to see similar data collected on larger and more representative samples of community-based primary care sites, including a broader sampling of primary care providers and public health departments in rural and medically underserved clinics, in order to derive more conclusive information on what providers need in terms of training and support to facilitate their provision of optimal health care to cancer patients.

The challenge is to continue improving the care of cancer patients in community care settings. We need to increase awareness about the vital role community-based primary care providers play in recognizing and actively addressing cancer-related problems, and to encourage and assist primary care providers in taking a central role in the continuum of care for cancer patients. To date there is very little data available on what is actually happening in post-treatment cancer care in community health care settings, or information on the training needs of primary care physicians and other types of health care providers working with cancer survivors. Our study provides some limited but useful data on these topics.

\section{References}

Ades, T., Alteri, R., Ganster, T., Kattlove, H., Luton, F., McClure, J., Pollack, A. and Rafine, S. 2002: Clinical practice guidelines in oncology, prostate cancer, version IV. National Comprehensive Cancer Network. Online at http//www.nccn.org (retrieved 30 March 2005).

Anderson, K.O., Richman, S.P., Hurley, J., Palos, G., Valero, V., Mendoza, T.R., Gning, I. and Cleeland, C.S. 2002: Cancer 
pain management among underserved minority outpatients: perceived needs and barriers to optimal control. Cancer 94, 2295-304.

Anthony, T., Simmang, C., Hyman, N., Buie, D., Kim, D., Cataldo, P., Orsay, C., Church, J., Otchy, D., Cohen, J., Perry, W.B., Dunn, G., Rafferty, J., Ellis, C.N., Rakinic, J., Fleshner, P., Stahl, T., Gregorcyk, S., Ternent, C., Kilkenny, J.W. and Whiteford, M. 2004: Practice parameters for the surveillance and follow-up of patients with colon and rectal cancer. Diseases of the Colon \& Rectum 47, 807-17.

Asch, D.A., Jedrziewski, M.K. and Christakis, N.A. 1997: Response rates to mail surveys published in medical journals. Journal of Clinical Epidemiology 50,1129-36.

Aziz, N.M. 2002: Cancer survivorship research: challenge and opportunity. Journal of Nutrition 132, 3494S-503S.

Brotzman, G.L. and Robertson, R.G. 1998: Role of the primary care physician after the diagnosis of cancer: the importance of a team approach. Primary Care 25, 401-06.

Carr, D., Goudas, L., Lawrence, D., Pirl, W., Lau, J., DeVine, D., Kupelnick, B. and Miller, K. 2002: Management of cancer symptoms: pain, depression, and fatigue. Evidence Report/Technology Assessment No. 61 (Prepared by the New England Medical Center Evidence-Based Practice Center under Contract No. 290-97-0019). AHRQ Publication No. 02-E032. Rockville, MD: Agency for Healthcare Research and Quality.

Centers for Disease Control and Prevention (CDC). 2004: A national action plan for cancer survivorship: advancing public health strategies. Online at http://www.laf.org/ Public_Health/overview.pdf (retrieved 30 March 2005).

Chaplin, J.M. and Morton, R.P. 1999: A prospective, longitudinal study of pain in head and neck cancer patients. Head \& Neck 2, 531-37.

Connaughton, D. 2001: FPs play important role after patient is diagnosed with cancer. Family practice report, October 6.

Davis, M.P. and Walsh, D. 2004: Epidemiology of cancer pain and factors influencing poor pain control. American Journal of Hospice and Palliative Care 21, 137-42.

Dillman, D.A. 1978: Mail and telephone surveys: the total design method. New York: Wiley.

Dow, K.H. 2003: Challenges and opportunities in cancer survivorship research. Oncology Nursing Forum 30, 455-69.

Grunfeld, E., Gray, A., Mant, D., Yudkin, P., Adewuyl-Dalton, R., Cole, D., Stewart, J., Fitzpatrick, R. and Vessey, M. 1999: Follow-up of breast cancer in primary care vs. specialist care: results of an economic evaluation. British Journal of Cancer 79, 1227-33.

Grunfeld, E., Mant, D., Yudkin, P., Adewuyi-Dalton, R., Cole, D., Stewart, J., Fitzpatrick, R. and Vessey, M.P.V. 1996: Routine follow-up of breast cancer in primary care: a randomized trial. British Medical Journal 313, 665-69.

Howe, H.L., Katterhagen, J.G., Yates, J. and Lehnerr, M. 1992: Urban-rural differences in the management of breast cancer. Cancer Causes Control 3, 533-39.

Institute of Medicine and Commission on Life Sciences. 1999: In Hewitt, M and Simone, J.V. editors, Ensuring quality cancer care. National Cancer Policy Board, National Research Council. Washington, DC: National Academy Press.

Jemal, A., Clegg, L.X., Ward, E., Ries, L.A.G., Wu, X., Jamison, P.M., Wingo, P.A., Howe, H.L., Anderson, R.N. and Edwards, B.K. 2004: Annual report to the nation on the status of cancer, 1975-2001, with a special feature regarding survival. Cancer 101, 3-27.

Kadan-Lottick, N.S., Robison, L.L., Gurney, J.G., Neglia, J.P., Yasui, Y., Hayashi, R., Hudson, M., Greenberg, M. and Mertens, A.C. 2002: Childhood cancer survivors' knowledge about their past diagnosis and treatment: childhood cancer survivor study. Journal of the American Medical Association 287, 1832-39.

Kattlove, H. and Winn, R.J. 2003: Ongoing care of patients after primary treatment for their cancer. CA-A Cancer Journal for clinicians 54, 172-96.

Liff, J.M., Chow, W.H. and Greenberg, R.S. 1991: Rural-urban differences in stage of diagnosis: possible relationship to cancer screening. Cancer 67, 1454-59.

National Institutes of Health. 2002: State-of-the-science statement 22: symptom management in cancer: pain, depression and fatigue. Online at http://consensus.nih.gov/ta/022/ 022_statement.htm (retrieved 30 March 2005). Final Statement, October 26, 2002.

Petzke, F., Radbruch, L., Zech, D., Loick, G. and Grond, S. 1999: Temporal presentation of chronic cancer pain: transitory pains on admission to a multidisciplinary pain clinic. Journal of Pain Symptom Management 17,391-401.

President's Cancer Panel 2003-2004. 2004: Living beyond cancer: finding a new balance. Online at http:/pcp.cancer.gov (retrieved May 2004). National Cancer Institute, National Institutes of Health, US Department of Health \& Human Services.

Smith, T.J., Davidson, N.E., Schapira, D.V., Grunfeld, E., Muss, H.B., Vogel, V.G. and Somerfield, M.R. 1999: American society of clinical oncology 1998 update of recommended breast cancer surveillance guidelines. Journal of Clinical Oncology 17, 1080-82.

Wang, L. 2002: Cancer survivors still struggle to get adequate follow-up care. Journal of the National Cancer Institute 94, 714-15.

World Health Organization. 1996: Cancer pain relief: with a guide to opioid availability. Geneva, Switzerland: WHO Press. 